\title{
Predictive model for major complications after extensive abdominal surgery in primary advanced ovarian cancer
}

This article was published in the following Dove Medical Press journal: International Journal of Women's Health

\author{
Antoni Llueca ${ }^{1-3}$ \\ Anna Serra ${ }^{1-3}$ \\ Karina Maiocchi ${ }^{2,4}$ \\ Katty Delgado ${ }^{2,5}$ \\ Rosa Jativa ${ }^{2,6}$ \\ Luis Gomez ${ }^{2,4}$ \\ Javier Escrig ${ }^{3-5}$ \\ On behalf of the \\ MUAPOS working group \\ (Multidisciplinary Unit of \\ Abdominal Pelvic Oncology \\ Surgery) \\ 'Department of Obstetrics and \\ Gynaecology, University General \\ Hospital of Castellon, Castellon, \\ Spain; ${ }^{2}$ Multidisciplinary Unit \\ of Abdominal Pelvic Oncology \\ Surgery (MUAPOS), University \\ General Hospital of Castellon, \\ Castellon, Spain; ${ }^{3}$ Department of \\ Medicine, University Jaume I (UJI), \\ Castellon, Spain; ${ }^{4}$ Department of \\ General Surgery, University General \\ Hospital of Castellon, Castellon, \\ Spain; ${ }^{5}$ Department of Radiology, \\ ${ }^{6}$ Department of Anaesthesiology, \\ University General Hospital of \\ Castellon, Castellon, Spain
}

Correspondence: Antoni Llueca Multidisciplinary Unit of Abdominal Pelvic Oncology Surgery (MUAPOS), University General Hospital of Castellon, Av

Benicasim s/n, I2004, Castellon, Spain

Tel +34964726500

Email antonillueca@gmail.com
Background: Surgery for advanced ovarian cancer (AOC) frequently results in serious complications. The present study aimed to determine the importance of various factors and complications in cytoreductive surgery for AOC.

Patients and methods: The present study included 90 patients with AOC who underwent primary cytoreductive surgery in a single institution from January 2013 to August 2017. Demographic and clinicopathologic characteristics, surgical procedures, residual disease, and follow-up data were analyzed. Cytoreductive surgery was defined as complete (no residual tumor), optimal (residual tumor $<1 \mathrm{~cm}$ in diameter), and suboptimal (residual tumor $>1 \mathrm{~cm}$ in diameter). Grade III-IV complications were considered major. Patients were evaluated every 3-6 months. Results: Surgical outcome was complete in $75(82 \%)$, optimal in $5(6 \%)$, and suboptimal in 11 $(12 \%)$ patients. Major complications occurred in 28 (31\%) patients. Independent risk factors for major complications were $\geq$ five visceral resections, rectosigmoid resection, glissectomy, and pelvic peritonectomy. A score created by weighing the multivariate OR for each risk factor correctly predicted major complications in $67 \%$ of cases. A score cut-off of $>2$ discriminated between patients with and without complications in $79 \%$ of cases $(95 \% \mathrm{CI}: 70 \%-86 \%, P<0.001)$. Adjuvant chemotherapy was performed as planned in 67 patients (74\%), including $50(75 \%)$ without major complications and 17 (25\%) with major complications.

Conclusion: Risk factors for major complications in cytoreductive surgery for AOC are $\geq$ five visceral resections, rectosigmoid resection, glissectomy, and pelvic peritonectomy. Our model predicts morbidity based on major and minor classifications of complications.

Keywords: advanced ovarian cancer, debulking surgery, peritoneal cancer index, residual tumor, complications, predictive model, carcinomatosis, cytoreductive surgery, morbidity

\section{Introduction}

Advanced ovarian cancer (AOC) surgery aims to achieve maximal cytoreduction in order to increase survival and even provide a definitive cure in some cases. Generally, this comprises a very aggressive surgery that frequently results in serious postoperative complications, including patient mortality or the impossibility of administration of subsequent oncological treatments; these complications can directly influence survival. ${ }^{1}$ It is difficult to analyze the influence of these postoperative complications due to the lack of definition and standardization in the literature, which creates enormous variability in the results across the various studies published to date; this variability also manifests itself in the various predictive models available. ${ }^{2}$

To evaluate the effect of complications of AOC surgery, several recent studies have attempted to systematize the collection of data on postoperative complications 
and classify their importance in a clear and reproducible way. Some of these classification systems are based on the intensity of the complication itself (eg, the National Cancer Institute's patient-reported outcomes version of the common terminology criteria for adverse events), ${ }^{3}$ while others are based on the complexity of the treatment required (eg, the Clavien-Dindo, Accordion, and Memorial Sloan Kettering Cancer Center [MSKCC] grading systems). ${ }^{4-6}$ No classification system has yet proven to be definitively more useful and better adjusted than the others.

Greater complexity of a classification system can improve the precision in stratifying the severity of complications, but this comes at the expense of an increase in the dispersion in the information referring to the individual influence of the various factors involved. Our objective was to analyze whether the grouping of complications by severity in the MSKCC Secondary Events Grading System ${ }^{5}$ is sufficiently useful to discriminate the importance of various factors and types of postoperative complications in cytoreductive surgery for AOC and to evaluate its consequences on the later course of the disease.

\section{Patients and methods}

The present study included patients with stage IIIc and IV ovarian, peritoneal, and fallopian tube carcinoma, who underwent primary cytoreductive surgery at the Multidisciplinary Unit of Abdominal Pelvic Oncology Surgery (MUAPOS) of the University General Hospital of Castellon (Spain) from January 2013 to August 2017. The risk of suboptimal cytoreductive surgery (SCS) was evaluated using the radiologiclaparoscopic criteria for unresectability, and the MUAPOS guide to managing $\mathrm{AOC}$ was applied in the preoperative examination. ${ }^{7}$ Age and health status were also taken into account, and all patients had an Eastern Cooperative Oncology Group $(\mathrm{ECOG})^{8}$ score $<3$. All procedures were carried out by the same surgical team. Information on the patients' clinical and pathological characteristics, surgical procedures, and residual disease at surgery were collected prospectively and analyzed retrospectively.

Demographic, clinicopathologic, and follow-up data were analyzed. Patients who received neoadjuvant chemotherapy were excluded. All patients were staged in accordance with the International Federation of Obstetrics and Gynecology (FIGO) staging system. ${ }^{9}$ The Peritoneal Cancer Index (PCI) was used to quantitatively assess cancer distribution in the peritoneum, based on the calculation of the sizes of the lesions in 13 abdominopelvic regions. The sizes of the lesions were then converted to scores of 0-3: a lesion size score (LSS) of 0 indicates no visible tumor burden in the peritoneum, while LSSs of 1, 2, and 3 describe lesions with a maximum diameter of $0.5,5.0$, and $>5 \mathrm{~cm}$ or confluence of lesions, respectively. The PCI was calculated by summarizing the LSSs for all regions, giving a maximum $\mathrm{PCI}^{10}$ of 39 $(13 \times 3)$. The PCI was determined in all patients in the current study on preoperative thoracoabdominal computed tomography (CT) and/or laparoscopy. To quantify the radiological PCI, the largest tumor in the assessed region was chosen and assigned a score of $0-3$. The sum of the scores for each region was then used to calculate the radiological PCI. The PCI was calculated pre- and intraoperatively and was categorized into two ordinal levels: $1-10$ and $>10$.

Although the principal reference for cytoreductive surgery was defined in the consensus statement for colonic cancer, ${ }^{10}$ nowadays some authors are more restrictive with respect to the presence of residual tumor after AOC surgery. ${ }^{11}$ Complete cytoreductive surgery was defined as no residual macroscopic tumor, optimal cytoreductive surgery (OCS) as a residual tumor $<1 \mathrm{~cm}$ in diameter, and SCS as a residual tumor $>1 \mathrm{~cm}$ in diameter.

For the purpose of the present study, complications of grades I-II and III-IV were categorized as minor and major, respectively, in accordance with the MSKCC Surgical Secondary Events Grading System. ${ }^{5}$ When collecting data regarding complications, procedures that were cancer related (ie, diagnostic thoracocentesis for staging) were not considered.

Follow-up of patients started at the time of diagnosis. All patients received first-line adjuvant chemotherapy comprising six to eight cycles of intravenous carboplatin and paclitaxel. After primary adjuvant chemotherapy, the patients were evaluated every 3-6 months. The occurrence of relapse and the response to first-line chemotherapy were evaluated in accordance with response evaluation criteria in solid tumors. ${ }^{12}$

Written informed consent was provided by the patients, and the study was approved by the Ethics and Clinical Research Committee of the University General Hospital of Castellon. All procedures performed in this study were in accordance with the ethical standards of our institution and with the 1964 Helsinki declaration.

\section{Statistical analysis}

The variables were summarized in accordance with their nature using the median and IQR, or as frequencies and percentages. Univariate analysis was performed with the Mann-Whitney test or the Fisher's exact test. Multivariate analysis was performed by logistic binary regression, with major complications as the dependent variable and the statistically significant factors in the univariate analysis as independent variables. A score was calculated for each independent risk factor from the multivariate OR. Finally, 
the area under the receiver operating characteristic (ROC) curve, sensitivity, specificity, and predictive values of the total score for each patient were calculated in accordance with the scores of the independent risk factors. Statistical analyses were performed using MedCalc 15 for Windows (version 15; MedCalc Software, Ostend, Belgium). For all univariate tests, a $P$-value $<0.05$ was considered statistically significant, and a more conservative $P$-value $\leq 0.10$ was considered to indicate the most important risk factors in multivariate regression.

\section{Results}

During the study period, 90 patients met the inclusion criteria.

Table 1 summarizes the clinicopathologic characteristics

Table I Clinicopathological characteristics of all patients

\begin{tabular}{|c|c|}
\hline Characteristics & Total series $(n=90)$ \\
\hline \multicolumn{2}{|l|}{ Age groups (years) } \\
\hline$\leq 60$ & $42(47 \%)$ \\
\hline$>60$ & $48(53 \%)$ \\
\hline \multicolumn{2}{|l|}{ FIGO stage } \\
\hline III & 64 (7I\%) \\
\hline IV & $26(29 \%)$ \\
\hline \multicolumn{2}{|l|}{ Histology } \\
\hline Serous & $7 \mathrm{7l}(79 \%)$ \\
\hline Mucinous & $6(7 \%)$ \\
\hline Endometrioid/clear cells & $13(14 \%)$ \\
\hline \multicolumn{2}{|l|}{$\mathrm{AACl}$ category } \\
\hline $\mathrm{AACl} 0$ & $35(39 \%)$ \\
\hline $\mathrm{AACl} \mathrm{I}-3$ & $25(28 \%)$ \\
\hline $\mathrm{AACl} 4-8$ & $30(33 \%)$ \\
\hline \multicolumn{2}{|l|}{ Computed tomography $\mathrm{PCl}$ category } \\
\hline $1-10$ & 47 (52\%) \\
\hline$>10$ & 43 (48\%) \\
\hline CEA* & I.3 (0.8-2) \\
\hline CA199* & $15.9(3-38)$ \\
\hline CAI25* & $197(58-638)$ \\
\hline CAI53* & $34(16-101)$ \\
\hline \multicolumn{2}{|l|}{ Laparoscopic PCl category } \\
\hline $1-10$ & $22(24 \%)$ \\
\hline$>10$ & $68(76 \%)$ \\
\hline \multicolumn{2}{|l|}{ Operative $\mathrm{PCl}$ category } \\
\hline $1-10$ & 40 (44\%) \\
\hline$>10$ & $50(56 \%)$ \\
\hline
\end{tabular}

(Continued)
Table I (Continued)

\begin{tabular}{|l|l|}
\hline Characteristics & Total series (n=90) \\
\hline Number of visceral resections & \\
0 & $12(13 \%)$ \\
1 & $17(19 \%)$ \\
2 & $19(21 \%)$ \\
\hline 3 & $11(12 \%)$ \\
4 & $6(7 \%)$ \\
5 & $7(8 \%)$ \\
6 & $5(6 \%)$ \\
7 & $3(3 \%)$ \\
8 & $5(6 \%)$ \\
9 & $1(1 \%)$ \\
II & $2(2 \%)$ \\
I3 & $1(1 \%)$ \\
I4 & $1(1 \%)$ \\
\hline Visceral resections & \\
Rectum & \\
Colon & $29(32 \%)$ \\
Bowel & $66(73 \%)$ \\
Urinary bladder & $23(26 \%)$ \\
Ureter & $14(16 \%)$ \\
Stomach & $5(6 \%)$ \\
Pancreas & $6(7 \%)$ \\
Spleen plus pancreas & $8(9 \%)$ \\
Gallbladder & $26(29 \%)$ \\
Liver & $22(24 \%)$ \\
Glisson & $6(7 \%)$ \\
Diaphragm & $15(17 \%)$ \\
Major vessels & $12(13 \%)$ \\
\hline Residual tumor & $3(3 \%)$ \\
CCS & \\
OCS & $17(11-31)$ \\
SCS & $74(82 \%)$ \\
\hline Postoperative stay* & $5(6 \%)$ \\
\hline Note & \\
\hline & \\
\hline & \\
\hline & \\
\hline & \\
\hline
\end{tabular}

Note: *Median (IQR).

Abbreviations: $\mathrm{AACl}$, Age-adjusted Charlson Comorbidity Index; $\mathrm{CA}$, carbohydrate antigen; CCS, complete cytoreductive surgery (no residual macroscopic tumor); CEA, carcinoembryonic antigen; FIGO, International Federation of Obstetrics and Gynecology; OCS, optimal cytoreductive surgery (residual tumor $<\mathrm{I} \mathrm{cm}$ in diameter); $\mathrm{PCl}$, Peritoneal Cancer Index; SCS, suboptimal cytoreductive surgery (residual tumor $>\mathrm{I} \mathrm{cm}$ in diameter).

of the included patients. The median age was 63 years, median Ca 125 was $197 \mathrm{U} / \mathrm{mL}$, number (percentage) of patients with FIGO stage III was 64 (73\%), median PCI on CT was 9, and serous histology was present in 71 patients (79\%). The cytoreductive surgery outcome was complete in 74 patients (82\%), optimal in $5(6 \%)$, and suboptimal in 11 patients $(12 \%)$.

Table 2 lists the principal factors for major complications. There were 247 upper abdominal procedures performed in the 90 patients. The most common procedures were pelvic peritonectomy, major omentectomy, and right diaphragm peritonectomy, which were performed in $61(67 \%), 69$ 
Table 2 Main prognostic factors for major complications

\begin{tabular}{|c|c|c|c|c|}
\hline & $\begin{array}{l}\text { Total series } \\
(\mathbf{N}=90)\end{array}$ & $\begin{array}{l}\text { No/minor complications } \\
(n=62)\end{array}$ & $\begin{array}{l}\text { Major complications } \\
(\mathrm{n}=\mathbf{2 8})\end{array}$ & $P$-value ${ }^{a}$ \\
\hline Computed tomography $\mathrm{PCl}$ category & & & & 0.042 \\
\hline $1-10$ & 47 (52\%) & 37 (60\%) & $10(36 \%)$ & \\
\hline$>10$ & $43(48 \%)$ & $25(40 \%)$ & $18(64 \%)$ & \\
\hline Operative $\mathrm{PCl}$ category & & & & 0.005 \\
\hline $1-10$ & 40 (44\%) & 34 (55\%) & $6(21 \%)$ & \\
\hline$>10$ & $50(56 \%)$ & $28(45 \%)$ & $22(79 \%)$ & \\
\hline Rectal resection & $29(32 \%)$ & $15(24 \%)$ & $14(50 \%)$ & 0.027 \\
\hline Sigmoid resection & 37 (4l\%) & $20(32 \%)$ & 17 (6I\%) & 0.020 \\
\hline Combined rectosigmoid resection & $22(24 \%)$ & $10(16 \%)$ & $12(43 \%)$ & 0.009 \\
\hline Spleen-distal pancreas resection & $26(29 \%)$ & $12(19 \%)$ & $14(50 \%)$ & 0.005 \\
\hline Glissectomy & $15(17 \%)$ & $6(10 \%)$ & $9(32 \%)$ & 0.013 \\
\hline Number of visceral resections & & & & 0.002 \\
\hline$<5$ & $65(72 \%)$ & $5 \mathrm{I}(82 \%)$ & 14 (50\%) & \\
\hline$\geq 5$ & $25(28 \%)$ & II (I8\%) & $14(50 \%)$ & \\
\hline Peritonectomy & & & & 0.017 \\
\hline None & $14(16 \%)$ & $9(15 \%)$ & $5(18 \%)$ & \\
\hline Partial & 48 (53\%) & 39 (63\%) & $9(32 \%)$ & \\
\hline Complete & $28(31 \%)$ & $14(23 \%)$ & $14(50 \%)$ & \\
\hline Pelvic peritonectomy & $60(67 \%)$ & $38(61 \%)$ & 22 (79\%) & 0.15 \\
\hline
\end{tabular}

Note: 'Fisher's exact test.

Abbreviation: $\mathrm{PCl}$, Peritoneal Cancer Index.

(76\%), and $44(48 \%)$ patients, respectively. Pelvic peritonectomy included common procedures such as hysterectomy and bilateral salpingo-oophorectomy. Pelvic and aortic lymphadenectomy was performed in 51 patients $(56 \%)$. Visceral resections were performed in 78 patients $(86 \%)$, with 25 patients (27\%) undergoing five or more resections. The median estimated blood loss was $1,600 \mathrm{~mL}$, with a median surgical time of 370 minutes.

Major complications occurred in 28 patients (31\%). Of the 28 patients with major complications, 17 cases $(61 \%)$ were managed by placing percutaneous or transvaginal drains for abdominal collections. Digestive leakages were found in ten patients $(11 \%)$, and colorectal leakages occurred in three patients $(3 \%)$.

The 30-day postoperative mortality rate was $2 \%$. Logistic regression analysis identified four independent risk factors for major complications: five or more visceral resections, combined rectal and sigmoid resection, glissectomy, and pelvic peritonectomy. A predictive score for major complications was built through weighing the multivariate OR for each independent risk factor (Table 3).

The scores of each patient correctly predicted the occurrence of major complications in $67 \%$ of cases. The calibration and discrimination power of the model was validated using the same data from the series (narrow validation). For an optimal score cut-off of $>2$, the discrimination between patients with and without complications was $79 \%$ (area under the ROC curve, $95 \%$ CI: $70 \%-86 \%, P<0.001)$. The sensitivity was $68 \%$, and the specificity was $82 \%$. For a general prevalence of major complications of $31 \%$, the positive predictive value was $63 \%$ and the negative predictive value was $85 \%$. Calibration of the model was assessed by comparing the predicted probabilities estimated from the predictive model with the actual observed proportion of patients with the outcome of interest (Figure 1).

The postoperative adjuvant chemotherapy plan for the 90 patients was to treat them with platinum and taxane chemotherapy for at least six cycles within 8 weeks postoperatively. Of the 90 patients, 67 (74\%) were able to start the six cycles of chemotherapy on time. Twenty-three patients (26\%) did not complete the prescribed postoperative chemotherapy, which was due to death in 4 patients (including

Table 3 Multivariate analysis: logistic regression for the presence of major complications

\begin{tabular}{l|l|l|l}
\hline Risk factors for major complications & OR & $P$-value & Score \\
\hline Five or more visceral resections & 3.6 & 0.07 & 2 \\
Rectosigmoid resection & 3.1 & 0.02 & 1.5 \\
Glissonian peritonectomy & 4.3 & 0.09 & 2 \\
Pelvic peritonectomy & 2.2 & 0.10 & $\mathrm{I}$ \\
\hline
\end{tabular}




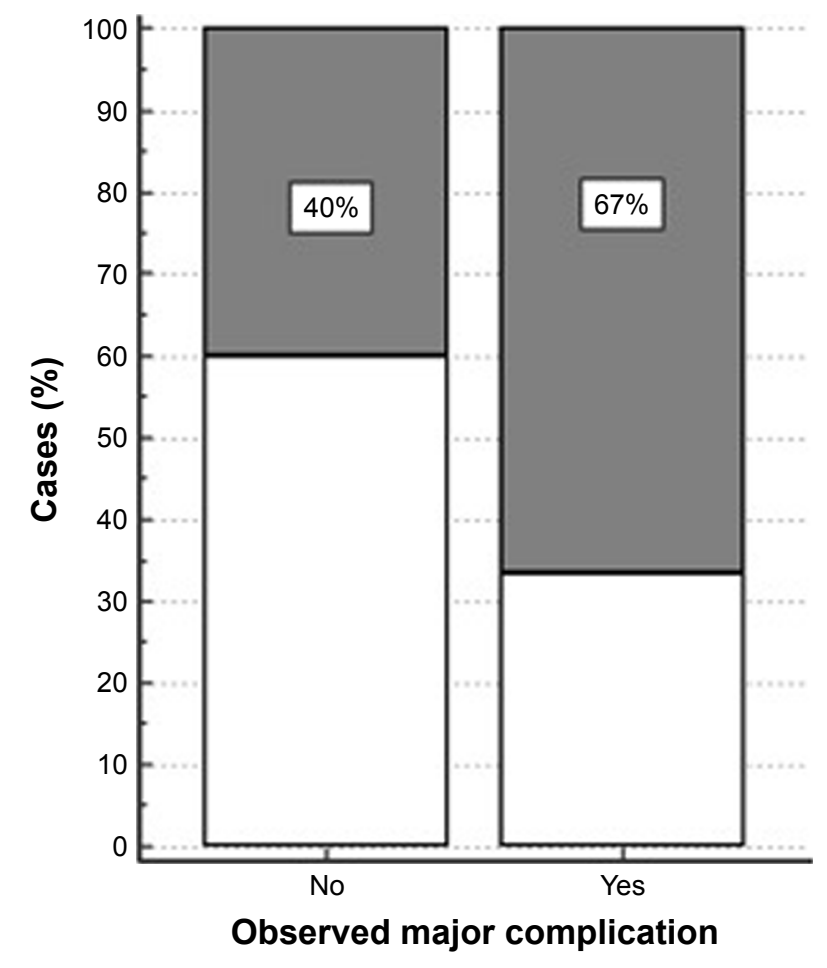

Predicted major complications

Yes (score >2) $\square$ No (score $\leq 2$ )

Figure I Calibration for an optimal score cut-off of $>2$.

the two postoperative mortalities) and the administration of chemotherapy outside of the planned time in 19 patients. Also, 81\% (50/62) of patients without major postoperative complications received adjuvant treatment, compared with $60 \%(17 / 28)$ with major complications.

Patients with a score $>2$ showed a trend toward worse overall survival $(\mathrm{HR}=1.6, P=0.10$; Figure 2$)$.

\section{Discussion}

Morbidity and mortality are generally considered the principal endpoints in AOC surgery. Our results suggest that the most important risk factors for major complications in AOC surgery were $>5$ visceral resections, rectosigmoid resection, and Glissonian and pelvic peritonectomy. The proposed model predicted these complications in nearly $70 \%$ of patients in our series.

In 2013, upper abdominal surgical (UAS) techniques were incorporated into the cytoreductive approach for advanced ovarian, fallopian, and peritoneal carcinomas. ${ }^{13}$ This led to a significant improvement in optimal cytoreductive rates, and improved overall and progression-free survival. ${ }^{7}$ Previous research reported that UAS techniques were required to achieve OCS in a large proportion of patients with AOC. ${ }^{14}$ Requirement of UAS techniques is not currently

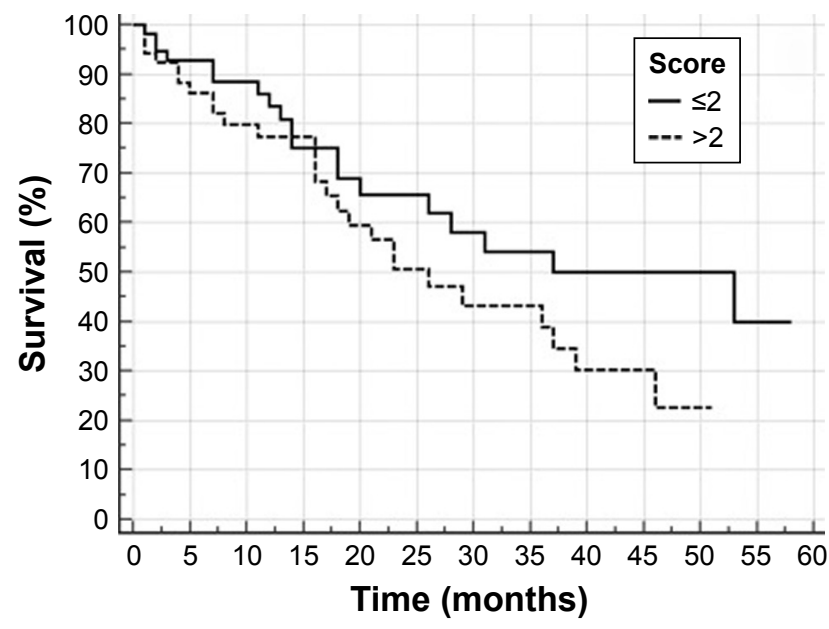

Figure 2 Overall survival in accordance with the score for major complications.

considered a contraindication to primary debulking surgery (PDS) in centers with an aggressive surgical protocol and higher rates of OCS. ${ }^{14,15}$

While previous studies have evaluated the advantages of incorporating these surgical techniques in terms of achieving OCS, ${ }^{14}$ the risk of complications associated with these procedures was not specifically evaluated. In the present study, we included patients who were optimally cytoreduced with standard surgical procedures and with UAS techniques, which could have led to a lowered complication rate. However, considering that UAS techniques had to be applied in nearly two-thirds of the patients in the present series, ${ }^{16}$ both standard techniques and UAS had to be considered together to accurately quantify morbidity and mortality associated with PDS in AOC.

Digestive anastomosis after intestinal resection is related to the occurrence of postoperative complications in patients undergoing extensive cytoreduction surgery. The anastomotic leakage rate after resection of a segment of the colon, especially in rectosigmoid resection, is reportedly $3 \%-15 \% .{ }^{17}$ In our series, dehiscence of the anastomosis occurred in ten patients (11\%), of which three (3\%) had undergone colorectal anastomosis. Performing protective ostomy after colorectal anastomosis is a controversial issue. ${ }^{18}$ Our group restricts the use of protective ostomy only to those patients requiring ultralow rectal resections or those in whom more than two intestinal resections were performed.

In the present study, we chose to focus on intraoperative factors, as surgical complexity is determined by the procedures performed during surgery. The next step could be the prediction of these surgical maneuvers using preoperative CT images, as described in other predictive models. ${ }^{2}$ Some institutions are now using laparoscopic assessments 
to determine resectability in patients with AOC. ${ }^{11}$ We have previously described a combination of preoperative CT and diagnostic laparoscopy to determine the tumor burden in AOC by calculating the PCI. ${ }^{16}$ The same previous study reported that patients with a $\mathrm{PCI}>20$ required three or more visceral resections. ${ }^{16}$ Using this preoperative combination of $\mathrm{CT}$ and laparoscopy to identify intraoperative risk factors for complications and mortality may be more useful for both clinicians and patients.

In the present study, the rate of major complications was $31 \%$ and the mortality rate was $2 \%$. This postoperative mortality rate after extensive cytoreductive surgery is in the same range as other individual and population-based studies that report an average mortality in the perioperative period of $2.5 \%-3.7 \%{ }^{19}$

Although half of the complications in the present study were managed with less aggressive techniques, these complications were directly related to the completeness of or adherence to adjuvant treatments. As reported in previous studies, ${ }^{20}$ we believe that major complications have a prognostic impact, indicating that a substantial delay in and/or omission of adjuvant therapy play a prognostic role. We defined the patients suitable for surgery as those with an ECOG score $<2$, but other authors have used a wider concept of frailty to assess the actual fitness of patients for surgery in order to prevent delaying the chemotherapy; ${ }^{21}$ this could be the reason why there were 18 patients in the present study in whom chemotherapy was performed outside of the planned timeframe.

The strengths of the present study include the homogeneous cohort of patients, and the single-institution uniform approach to care and inclusion of patients with AOC, making it relevant to those at the greatest risk of surgical morbidity and mortality.

The limitations of the present study include the retrospective design and the optimistic biased performance of our model performed via internal validation. However, we feel that including those common surgical factors performed in most AOC cytoreductive surgeries makes this model relevant in most settings. Nevertheless, external validation of this model in other settings is mandatory.

In conclusion, given the substantial survival benefit obtained from maximal cytoreduction, the management guidelines of AOC should allow a balanced approach to short-term morbidity and long-term benefit. Clinicians must always take into account that a greater degree of surgical aggression induces a greater number of serious complications, and these major complications may worsen the prognosis. We present a simple predictive model of morbidity based on major and minor classifications of complications, which will be useful in patient counseling and will aid clinicians and patients in making decisions regarding PDS. Some patients will be better managed using an alternative approach (neoadjuvant chemotherapy), but PDS is reasonable and well tolerated by the majority of patients. Further strategies to reduce surgical complications, plus referral of patients to experienced surgical teams should enable further reductions in morbidity and mortality.

\section{Acknowledgments}

Multidisciplinary Unit of Abdominal Pelvic Oncology Surgery Working Group includes the following: Gomez-Quiles L, Rivadulla I, Játiva-Porcar R, Moreno-Clarí E, MontañésPauls B, Granel-Villach L, Bellver M, Maiocchi K, Medina C, Delgado-Barriga K, Rodrigo-Aliaga M, Ruiz N, Lopez A, Maazouzi Y, Piquer D, Segarra B, and Del Moral R. We thank Kelly Zammit, BVSc, from Edanz Group for editing a draft of this manuscript. This work received financial support from the Medtronic University Chair for Training and Surgical Research, University Jaume I (UJI), Castellon, Spain.

\section{Disclosure}

The authors report no conflicts of interest in this work.

\section{References}

1. Vergote I, Tropé CG, Amant F, et al. Neoadjuvant chemotherapy or primary surgery in stage IIIC or IV ovarian cancer. $N$ Engl J Med Overseas Ed. 2010;363(10):943-953.

2. Kumar A, Janco JM, Mariani A, et al. Risk-prediction model of severe postoperative complications after primary debulking surgery for advanced ovarian cancer. Gynecol Oncol. 2016;140(1):15-21.

3. Basch E, Reeve BB, Mitchell SA, et al. Development of the National Cancer Institute's patient-reported outcomes version of the common terminology criteria for adverse events (PRO-CTCAE). J Natl Cancer Inst. 2014;106(9):dju244.

4. Clavien PA, Sanabria JR, Strasberg SM. Proposed classification of complications of surgery with examples of utility in cholecystectomy. Surgery. 1992;111(5):518-526.

5. Strong VE, Selby LV, Sovel M, et al. Development and assessment of Memorial Sloan Kettering cancer center's surgical secondary events grading system. Ann Surg Oncol. 2015;22(4):1061-1067.

6. Klos CL, Safar B, Hunt SR, et al. Accordion complication grading predicts short-term outcome after right colectomy. J Surg Res. 2014; 190(2):510-516.

7. Llueca A, Serra-Rubert A, Escrig J; MUAPOS Working Group (Multidisciplinary Unit of Abdominal Pelvic Oncology Surgery). Prognostic value of peritoneal cancer index in primary advanced ovarian cancer. Eur J Surg Oncol. 2018;44:163-169.

8. Oken MM, Creech RH, Tormey DC, et al. Toxicity and response criteria of the Eastern Cooperative Oncology Group. Am J Clin Oncol. 1982;5(6): 649-656.

9. Prat J; FIGO Committee on Gynecologic Oncology. Staging classification for cancer of the ovary, fallopian tube, and peritoneum. Int $J$ Gynecol Obstet. 2014;124:1-5.

10. Sugarbaker PH, Jablonski KA. Prognostic features of 51 colorectal and 130 appendiceal cancer patients with peritoneal carcinomatosis treated by cytoreductive surgery and intraperitoneal chemotherapy. Ann Surg. 1995;221(2):124-132. 
11. Petrillo M, Vizzielli G, Fanfani F, et al. Definition of a dynamic laparoscopic model for the prediction of incomplete cytoreduction in advanced epithelial ovarian cancer: proof of a concept. Gynecol Oncol. 2015;139(1):5-9.

12. Rustin GJ, Quinn M, Thigpen T, et al. Re: new guidelines to evaluate the response to treatment in solid tumors (ovarian cancer). JNatl Cancer Inst. 2004;96(6):487-488.

13. Llueca JA, Herraiz JL, Catala C, et al. Effectiveness and safety of cytoreduction surgery in advanced ovarian cancer: initial experience at a university general hospital. J Clin Gynecol Obstet. 2015;4(3):251-257.

14. Bristow RE, Tomacruz RS, Armstrong DK, Trimble EL, Montz FJ. Survival effect of maximal cytoreductive surgery for advanced ovarian carcinoma during the platinum era: a meta-analysis. J Clin Oncol. 2002;20(5):1248-1259.

15. Chi DS, Eisenhauer EL, Zivanovic O, et al. Improved progression-free and overall survival in advanced ovarian cancer as a result of a change in surgical paradigm. Gynecol Oncol. 2009;114(1):26-31.

16. Llueca A, Serra A, Rivadulla I, Gomez L, Escrig J; MUAPOS Working Group (Multidisciplinary Unit of Abdominal Pelvic Oncology Surgery). Prediction of suboptimal cytoreductive surgery in patients with advanced ovarian cancer based on preoperative and intraoperative determination of the peritoneal carcinomatosis index. World J Surg Oncol. 2018;16:37.
17. Gusani NJ, Cho SW, Colovos C, et al. Aggressive surgical management of peritoneal carcinomatosis with low mortality in a high-volume tertiary cancer center. Ann Surg Oncol. 2008;15(3):754-763.

18. Moran BJ, Cecil TD. The etiology, clinical presentation, and management of pseudomyxoma peritonei. Surg Oncol Clin N Am. 2003;12(3): 585-603.

19. Gerestein CG, Damhuis RA, Burger CW, Kooi GS. Postoperative mortality after primary cytoreductive surgery for advanced stage epithelial ovarian cancer: a systematic review. Gynecol Oncol. 2009;114(3): 523-527.

20. Vizzielli G, Costantini B, Tortorella L, et al. A laparoscopic riskadjusted model to predict major complications after primary debulking surgery in ovarian cancer: a single-institution assessment. Gynecol Oncol. 2016;142(1):19-24.

21. Feng MA, Mcmillan DT, Crowell K, Muss H, Nielsen ME, Smith AB. Geriatric assessment in surgical oncology: a systematic review. J Surg Res. 2015;193(1):265-272.
International Journal of Women's Health

\section{Publish your work in this journal}

The International Journal of Women's Health is an international, peerreviewed open-access journal publishing original research, reports, editorials, reviews and commentaries on all aspects of women's healthcare including gynecology, obstetrics, and breast cancer. The manuscript management system is completely online and includes

\section{Dovepress}

a very quick and fair peer-review system, which is all easy to use. Visit http://www.dovepress.com/testimonials.php to read real quotes from published authors.

\footnotetext{
Submit your manuscript here: http://www.dovepress.com/international-journal-of-womens-health-journal
} 